Urologe $2017 \cdot 56: 658$

DOI 10.1007/s00120-017-0377-5

Online publiziert: 19. April 2017

๑) Springer Medizin Verlag GmbH 2017

CrossMark

Winfried Vahlensieck

Chefarzt der Fachklinik Urologie, Kurpark-Klinik, Bad Nauheim, Deutschland

\title{
Zum Vorgehen bei Candidurie und der Nitroxolin-Empfindlich- keit von Candidaisolaten
}

In der Diskussion geht der Autor von einer Candidurierate von $3 \%$ in unselektionierten Urinen von ambulanten und stationären Patienten aus, obwohl in seiner eigenen Untersuchung (Tab. 1) lediglich eine Rate von $0,3 \%(380 / 128271$ Urinisolate) gefunden wurde, wobei die Keimzahl (KZ) unklar bleibt (Überschrift $\mathrm{KZ}>10^{4}$ (pro Milliliter?); Tabellentext KZ $\left.>10^{5} / \mathrm{ml}\right)$.

Mit Recht weist der Autor darauf hin, dass die klinische Relevanz eines Nachweises von Candida im Urin oft schwierig $\mathrm{zu}$ beurteilen ist. Auch aus diesem Grund ist eine Nachweisrate von $0,3 \%$ noch lange nicht mit einer Infektionsrate gleichzusetzen, da in seiner Untersuchung keine klinischen Angaben $\mathrm{zu}$ den untersuchten Isolaten vorliegen. Trotzdem erscheint die in vitro gemessene Empfindlichkeitsrate (nicht Wirksamkeitsrate!) von Nitroxolin mit $100 \%$ ermutigend. Eine Vergleichsuntersuchung gegenüber Standardantimykotika wäre zwar wünschenswert gewesen, aber die Untersuchung zeigt, dass zumindest aus mikrobiologischer Sicht Nitroxolin eine gute Alternative zu den Antimykotika für die Therapie einer Candidurie darstellen könnte, entsprechend dem Fazit des Autors für die Praxis.

\section{Korrespondenzadresse}

\section{PD Dr. W. Vahlensieck}

Chefarzt der Fachklinik Urologie, Kurpark-Klinik Kurstr. 41-45, 61231 Bad Nauheim,

Deutschland

Winfried.Vahlensieck@t-online.de

Interessenkonflikt. W. Vahlensieck gibt an, dass kein Interessenkonflikt besteht.

\section{Literatur}

1. Wagenlehner FM, Schmiemann G, Hoyme U, FünfstückR, Hummers-Pradier E, Kaase M, KniehIE, Selbach I, Sester U, Vahlensieck W, Watermann D, Naber KG (2010) S-3 Leitlinie Harnwegsinfektionen Epidemiologie, Diagnostik, Therapie und Management unkomplizierter bakterieller ambulant erworbener Harnwegsinfektionen bei erwachsenen Patienten. http://www.awmf.org/uploads/tx szleitlinien/043-044l_S3_Harnwegsinfektionen. pdf 